\title{
Aportaciones al estudio reológico de pastas y morteros de cemento portland
}

Fecha de recepción: 12-III-99

Fecha de aceptación: 8-VI-99

\author{
J.MIRANDA, Dpto. de Ingeniería Química. Univ. de Sevilla \\ V. FLORES-ALÉS, Dpto. de Construcciones Arquitectónicas II. Univ. de Sevilla \\ J. BARRIOS, Dpto. de Construcciones Arquitectónicas I. Univ. de Sevilla
}

ESPAÑA

\section{RESUMEN}

El hormigón se puede considerar como una suspensión de partículas sólidas gruesas en una suspensión de partículas finas. La reología de esta última influye sustancialmente sobre el comportamiento del hormigón, de ahi la importancia de realizar un estudio sobre la reología de pastas y morteros.

El primer objetivo de este trabajo ha consistido en determinar el tipo de reómetro más adecuado para caracterizar este tipo de muestras, considerando la dificultad que conlleva el estudio reológico de suspensiones de concentración elevada que tienden a desarrollar fenómenos de deslizamiento en las paredes del sistema sensor. Entre los equipos con sensor placa-placa, con cilindros coaxiles y con agitador de cinta helicoidal, fueron desechados los primeros debido a la alta relación diámetro de particula/hueco de medida que presentan y que hace que las propiedades viscosas sean escasamente repetitivas, mientras que el último aparato no presenta este problema, por lo que se optó por su utilización para la obtención de las propiedades reológicas de las muestras en estudio.

En segundo lugar, se ha tratado de describir el comportamiento viscoso de distintas pastas y morteros con diferentes agregados y relaciones agua/cemento. Para ello se realizaron barridos de velocidad de agitación y ensayos a velocidad constante, relacionando la evolución de la viscosidad con los periodos del fraguado.

El análisis del comportamiento reológico se ve afectado por el hecho de aplicar una agitación que provoca la ruptura de un sistema que tiende a estructurarse durante el proceso de fraguado, por lo que esta especificidad en función del tiempo, unida a la heterogeneidad de las muestras, dificulta la obtención de resultados con una buena repetibilidad.

\section{SUMMARY}

The concrete could be considered as a thik solid particles suspension in fine particles suspension. The rhealogy of the last one influences very hardly the concrete behaviour, so that the importance to realise an study concerning the mortars and pastes rheology.

The first objective of this work has been to determine the more adecuated rheometer to caracterize this kind of samples, considering the difficulty of the rheologic study of high concentration suspensions who tends towards the development of shear phenomena in the walls of the sensor system. Between the equipments with placa-placa sensor, coaxial cilinders and helicoidal band shaker, the first ones were discarted due to the high relationship between particle diameter and measure cavity that makes de viscose properties scarcely repetitives, whereas the last set doesn't present this problem, in view of which we choose to use it to the obtention of rheologic properties of the samples studied.

Secondly, we have try to describe the viscose behaviour of different pastes and mortars with different agregates and relations water/cement. For this purpose some sweeping of shake speed were realised as well as tests at constant speed, relating the viscosity evolution with the setting time.

The analyse of the rhealogic behaviour is affected by the fact of applying a shake that conduces to the system break who tends towards to be structured during the setting time. This specificity concerning the time, joined with the heterogeneity of the samples, makes difficult to obtain results with a good repetibility. 


\section{INTRODUCCIÓN}

La reología de pastas y morteros de cemento ha sido objeto de múltiples estudios. Sin embargo, este comportamiento no ha sido todavía totalmente explicado. De hecho, los datos existentes de diferentes autores son a menudo inconsistentes y contradictorios. Esto es debido, probablemente, al complejo comportamiento reológico de estos materiales, por la gran cantidad de factores de los que depende, y al desarrollo con el tiempo de los procesos de hidratación de los componentes del cemento.

El comportamiento y propiedades finales de un mortero dependen de numerosas variables como son:

-Calidad de cemento, arena, agua y aditivos usados.

-Proporciones de la mezcla.

-Forma, duración y temperatura del amasado.

-Condiciones de humedad y temperatura de su conservación.

La dosificación adecuada de un mortero debe ser tal que su comportamiento durante el período fluido y propiedades como resistencia a flexión y compresión, retracción y adherencia, sean las óptimas.

\section{Reología de pastas de cemento}

Las pastas presentan un comportamiento viscoplástico, con shear-thinning (fluido pseudoplástico) o sheartinckening (fluido dilatante), dependiendo del contenido en agua (1). Con una relación agua/cemento $(\mathrm{a} / \mathrm{c})$ en peso entre 0,32 y 0,40 , Papo (2) muestra, usando un viscosímetro de cilindros coaxiales, que estos fluidos se ajustan al modelo de Bingham o de Herchel-Bulkey. Sin embargo, este comportamiento no se da por debajo de una relación $\mathrm{a} / \mathrm{c}=0,32$.

Caufin y Papo (3), usando un viscosímetro de cilindros coaxiales (Rotovisco RV3, Haake), demostraron que cuando las pastas de cemento con una relación a/c entre 0,30 y 0,40 eran sometidas a ensayos a velocidad de cizalla constante mostraban un comportamiento no newtoniano dependiente del tiempo (Figura 1), caracterizado por:

-Un sobreesfuerzo inicial, $\tau_{p}$, que se corresponde con las estructuras obtenidas después del mezclado.

-Una caída del esfuerzo hasta un valor de equilibrio, $\tau_{e}$, debida al efecto de la rotura estructural tixotrópica causada por la cizalla.

-Un posterior incremento del esfuerzo debido a que el

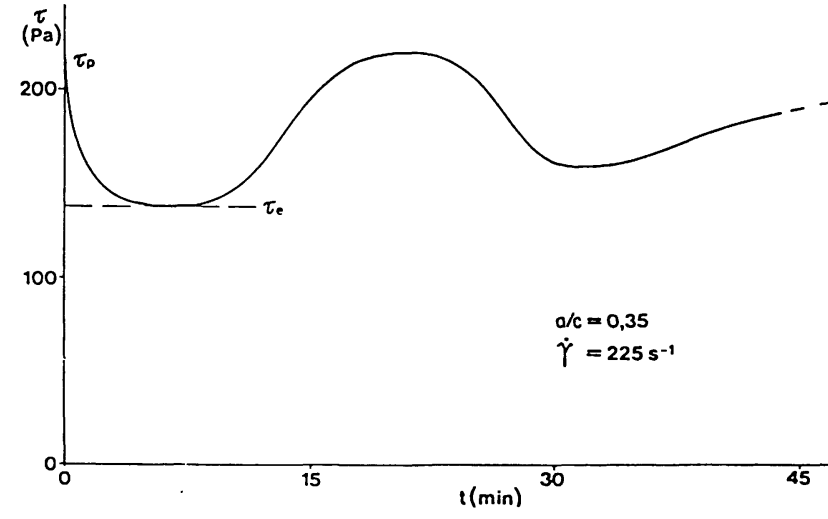

Figura 1.- Evolución del esfuerzo a velocidad de cizalla constante (225 $\mathrm{rad} / \mathrm{s}$ ) de la pasta de cemento $\mathrm{a} / \mathrm{c}=0,35$. Experiencia realizada por Caufin y Papo (3).

proceso de fraguado del cemento se impone al de rotura estructural por cizalla. En algunos casos, el esfuerzo alcanza un máximo después del cual desciende hasta un mínimo y posteriormente aumenta de forma gradual hacia valores muy altos.

\section{Reología de morteros de cemento}

Los ensayos reológicos sobre morteros de cemento son difíciles de llevar a cabo debido al tamaño de los áridos, por lo que se han de comparar diferentes sistemas de medida para poder definir el verdadero comportamiento reológico de las muestras.

Los morteros muestran destrucción estructural con la cizalla y su curva de flujo resultante se ajusta al modelo de Bingham, es decir, a un comportamiento plástico (4), de esta forma existe una relación lineal entre el torque o par torsor (M), que da idea de la consistencia del mortero, y la velocidad de agitación $(\mathrm{N})$ :

$$
\mathrm{M}=\mathrm{g}+\mathrm{hN}
$$

Este método usa parámetros globales como g, que es el torque umbral, y h, que está relacionado con la viscosidad plástica. En este caso el autor usó un mezclador con control de la velocidad de agitación y registro del par torsor (Visco-Corder, Brabender).

Recientemente, usando un viscosímetro de cilindros coaxiales, Costil y Chappuis (5) han demostrado el comportamiento tixotrópico de morteros en los cuales el volumen de sólidos es del $48 \%$.

Por otro lado, Wolter (6) realizó ensayos a velocidad de cizalla constante de morteros con una relación agua/ cemento/arena (a/c/a) de 1/2/3. La variación del esfuerzo con el tiempo es bastante similar a la mostrada por la pasta de cemento. En primer lugar, se da un descenso por efecto 
de la rotura estructural (tixotropía), seguido de una zona de transición donde el esfuerzo se mantiene prácticamente constante $y$, finalmente, una zona donde el esfuerzo aumenta debido a las reacciones de hidratación del fraguado.

\section{Microestructura de la pasta de cemento}

La microestructura de la pasta de cemento en estado fresco se basa en las uniones que se crean entre partículas sólidas que en el momento del primer contacto aguacemento se encontraban tocándose. Alrededor de las partículas se forma una capa de material hidratado que las mantiene unidas formando flóculos en forma de platos o de esferas (7).

El endurecimiento de la pasta de cemento Portland y su acción cementante es resultado esencialmente de la formación del gel de tobermorita (8), pues las pastas preparadas con los silicatos tricálcico y bicálcico fraguan y endurecen de igual forma que las preparadas con cemento Portland y las resistencias finales desarrolladas por ambas fases minerales son prácticamente iguales.

Asimismo, el proceso de hidratación o fraguado de los cementos da lugar a la formación de estructuras complejas, integradas también por diversas especies minerales hidratadas que conforman básicamente un gel formado por partículas microcristalinas de dimensiones coloidales que determinan, en gran parte, las propiedades de la roca de cemento endurecida (9).

El problema de la estructura de la pasta de cemento endurecida hace surgir cada vez el contraste entre la hipótesis de Le Chatelier (10), sobre la naturaleza cristalina de aquélla, y la de Michaëlis (11), sobre el carácter de gel de la misma. Actualmente, se acepta una síntesis de ambas, al ser considerada la estructura como un gel formado de partículas microcristalinas de tobermorita, las cuales, en determinadas circunstancias, pueden aumentar su grado de cristalización.

La formación de la estructura del gel microcristalino define las resistencias mecánicas debidas al proceso de hidratación de los cementos en los morteros, estructura que comienza a formarse desde el inicio de la reacción, aunque la manifestación de la resistencia mecánica implica la presencia de un período de inducción (12), tornándose cada vez más densa y compacta.

En el caso de los morteros, la complejidad se acentúa grandemente, dado que el cemento se hidrata en presencia de otras sustancias minerales, tales como los áridos, que aportan sus propias características mineralógicas, granulométricas y morfológicas. Además, hay que considerar la adición de cantidades de agua variables, así como el procedimiento de preparación y ulterior curado del mortero.

El hidrogel ideal es así modificado por la introducción de una fase sólida heterogénea. Cuando el tamaño de partícula del sólido es superior al espacio máximo intercristalino en el gel, este último puede ser considerado como un medio de suspensión de los áridos.

\section{MATERIALES Y MÉTODOS}

\section{Materíales y preparación de muestras}

Los productos utilizados fueron cemento Portland CEM II/A-L 32,5 de Cementos Atlántico (Valenciana de Cementos), arena convencional y agua destilada. El CEM II/ A-L 32,5 es un cemento Portland cuya composición según la RC-97 (13) debe estar comprendida entre: 80 $94 \%$ de clínker, $6-20 \%$ de caliza y $0-5 \%$ de componentes minoritarios adicionales. Mientras que la arena usada fue de naturaleza mayoritariamente silícea con fracción caliza del $4 \%$ en peso.

Para la preparación de los morteros se tamizó una arena convencional con el fin de obtener curvas granulométricas dentro de los márgenes que según la NBE FL90 (14) proporcionan las mejores propiedades del mortero una vez fraguado. Además, se usó arena normalizada (UNE 80-101-91), suministrada por el Instituto de Ciencias de la Construcción Eduardo Torroja, cuya curva granulométrica se representa, junto con los límites superior e inferior de la FL90 y una línea intermedia, en la Figura 2.

Respecto a las pastas de cemento, las relaciones agua/ cemento $(\mathrm{a} / \mathrm{c})$ estudiadas fueron 0,$26 ; 0,35 ; 0,45$ y 0,50 . Por otro lado, se estudiaron morteros con una proporción agua/cemento/arena (a/c/an) de 1/2/2

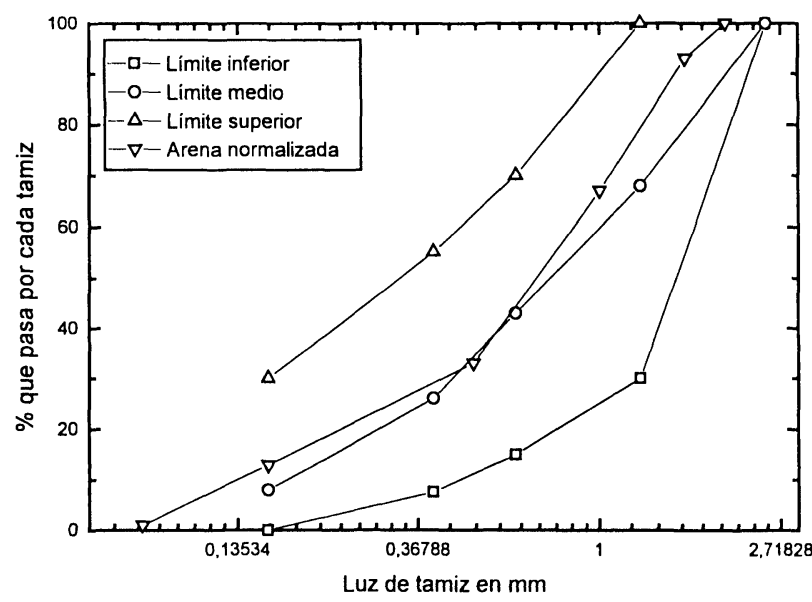

Figura 2.- Curvas granulométricas de las arenas utilizadas 
Tanto la pasta como los morteros fueron preparadas en una amasadora normalizada (UNE-101-88). El protocolo de amasado seguido en cada caso se detalla en la Tabla 1.

\section{Reómetros utilizados}

El comportamiento reológico de las pastas y morteros de cemento es bastante complejo. Esto, unido a que el tamaño de partículas de las arenas, en el caso de los morteros, es demasiado grande para poder utilizar los sistemas sensores tradicionales (16) (cilindros coaxiales, placa-placa o cono-placa), hace necesario un estudio previo para determinar que reómetro es el más adecuado para caracterizar estos materiales. Para resolver este problema con una cierta seguridad y eficacia se realizó una amplia búsqueda bibliográfica sobre el comportamiento reológico tanto de pastas de cemento como de morteros, así como los reómetros utilizados por diferentes autores (Tabla 2).
Para el estudio de las propiedades viscosas de la pasta de cemento se usaron 4 tipos de reómetros diferentes:

- Reómetro de esfuerzo controlado:Haake (Karlsruhe, Alemania) RS100, con sistema sensor placa-placa de superficie rugosa.

- Reómetros de velocidad controlada: Haake RV-CV20N con sistema sensor placa-placa rugoso; Haake RV-CV100 con sistema sensor placa-placa rugoso; Ika (Stäufen, Alemania) MR-D1 con agitador de cinta helicoidal.

Para realizar los ensayos sobre los morteros únicamente se usó el mezclador convencional Ika MR-D1, con control de velocidad de agitación y lectura del par torsor. Se empleó un agitador de cinta helicoidal, de $13 \mathrm{~cm}$ de altura y $12 \mathrm{~cm}$ de diámetro, montado dentro de un vaso cilíndrico de $14,8 \mathrm{~cm}$ de diámetro interno y $18,5 \mathrm{~cm}$ de altura.

TABLA 1

Protocolos de amasado de las pastas y morteros de cemento

\begin{tabular}{|c|c|c|}
\hline TIEMPO (MIN) & MORTERO & PASTA \\
\hline 0 & $\begin{array}{c}\text { Agua en amasadora, adición de cemento, } \\
\text { velocidad lenta. }\end{array}$ & $\begin{array}{c}\text { Agua en amasadora, mitad de cemento, } \\
\text { velocidad lenta. }\end{array}$ \\
\hline 2 & Adición de arena, velocidad lenta. & $\begin{array}{c}\text { Adición de resto de cemento, velocidad } \\
\text { lenta. }\end{array}$ \\
\hline 4 & Velocidad lenta. & Velocidad lenta. \\
\hline 6 & Reposo & Reposo \\
\hline 7,5 & Velocidad lenta. & Fin. \\
\hline 10 & Fin. & Velocidad lenta. \\
\hline
\end{tabular}

TABLA 2

Reómetros utilizados para pasta y morteros de cemento por diferentes autores

\begin{tabular}{||c|c|c|c|}
\hline \multicolumn{2}{|c|}{ PASTA } & REÓMETRO & RORTO \\
\hline REÓMETRO & AUTOR & ViscoCorder \\
\hline $\begin{array}{c}\text { Strathclycle Rheometer } \\
\text { (mezclador de paleta) } \\
\text { (mezclador de paleta) }\end{array}$ & Panfill y cols. (17) \\
\hline $\begin{array}{c}\text { Rotovisco RV3 Haake } \\
\text { (cilíndros coaxiles) }\end{array}$ & Caufin y Papo (3) & BuTOR \\
\hline $\begin{array}{c}\text { VT500 Haake } \\
\text { (rotacional) }\end{array}$ & Wang (15) & $\begin{array}{c}\text { ViscoCorder } \\
\text { (mezclador de paleta) }\end{array}$ & G. Wolter (6) \\
\hline
\end{tabular}


Se realizaron tanto barridos de velocidad de cizalla y de velocidad de agitación, como ensayos a una velocidad constante para estudiar el proceso de hidratación de la pasta de cemento. Los ensayos realizados en el mezclador Ika constan de un barrido de velocidades en forma de ciclo de ida y vuelta tanto al principio como al final de la experiencia y en una zona intermedia donde se mantiene una velocidad constante de $50 \mathrm{rev} / \mathrm{min}$ (Figura 3 ).

\section{RESULTADOS Y DISCUSIÓN}

Se realizaron ensayos reológicos con diferentes tipos de reómetros, a fin de determinar qué aparato era el más adecuado para caracterizar la pasta de cemento y, además, obtener ciertas conclusiones sobre el sistema a utilizar con los morteros.

Se llevaron a cabo experiencias sobre la pasta de cemento $\mathrm{a} / \mathrm{c}=0,40$, con el reómetro RS100, llegándose a la conclusión de que éste no tenía la fuerza necesaria para cizallar dicha pasta y menos aún los morteros.

En la Figura 4 pueden observarse las curvas de flujo obtenidas mediante ensayos realizados en los reómetros CV20 y CV100 que demuestran, en el intervalo de velocidades de cizalla estudiado, el comportamiento pseudoplástico de la pasta de cemento con unas relaciones a/c de 0,26,0,35 y 0,45. Además, se observa un aumento de la viscosidad con la disminución de la relación $\mathrm{a} / \mathrm{c}$. Por otro lado, estas pastas de cemento muestran un comportamiento tixotrópico al someterlas a un ensayo a velocidad de cizalla constante (Figura 5). En principio existe un sobreesfuerzo característico de la estructura inicial de la pasta, seguido de una disminución debido a la destrucción estructural por cizalla. Este efecto se ve contrarrestado por el aumento de la estructuración debida al proceso de fraguado que provoca un aumento del esfuerzo. Aunque esta nueva estructura, creada por las reacciones de hidratación, puede ser destruida por la cizalla, provocando otra caída del esfuerzo, $\tau$. Esta competencia de fuerzas, fraguado y cizalla, puede dar lugar a una oscilación del esfuerzo de cizalla con el tiempo. Sin embargo, los dos tipos de ensayos realizados en los reómetros CV20 y CV100, flujo en estado estacionario y ensayos a velocidad de cizalla constante, demostraron la escasa repetibilidad de las propiedades reológicas de suspensiones concentradas en los reómetros tradicionales.

En la Figura 4 se representan tres replicados de las curvas de viscosidad realizados en el reómetro CV20 para la pasta de cemento con relación a/c de 0,26 . Se llevó a cabo un análisis estadístico para determinar la repetibilidad del ensayo, observándose que dos de los replicados están fuera de los límites de confianza para el $95 \%$ de probabilidad y que la media del error cometido, para cada velocidad de cizalla, está en torno al $37 \%$.

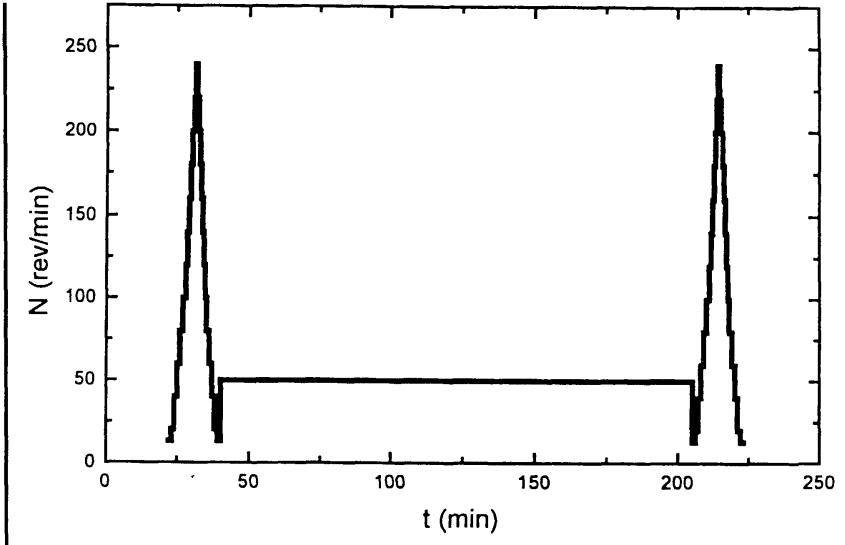

Figura 3.- Experiencias realizadas en el agitador IKA MR-D1.

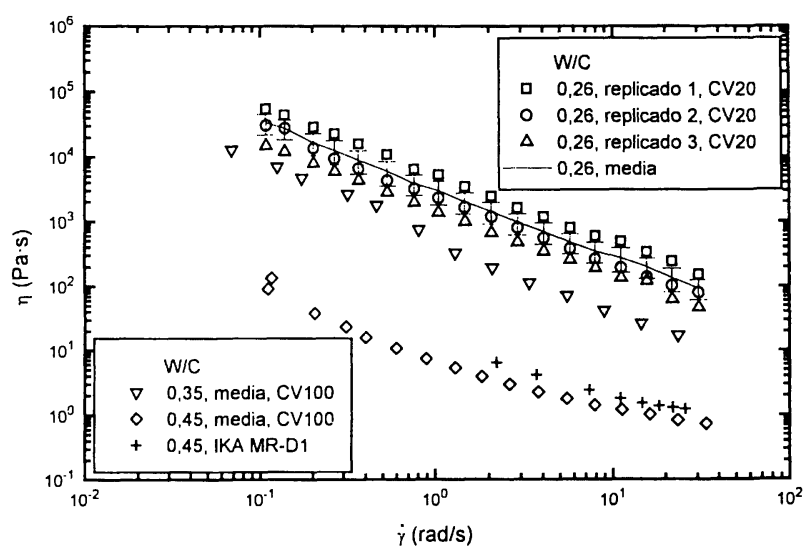

Figura 4.- Curvas de flujo realizadas en los reómetros CV20 y CV100, y en el mezclador convencional IKA MR-D1, para pastas de cemento a diferentes relaciones agua/cemento.

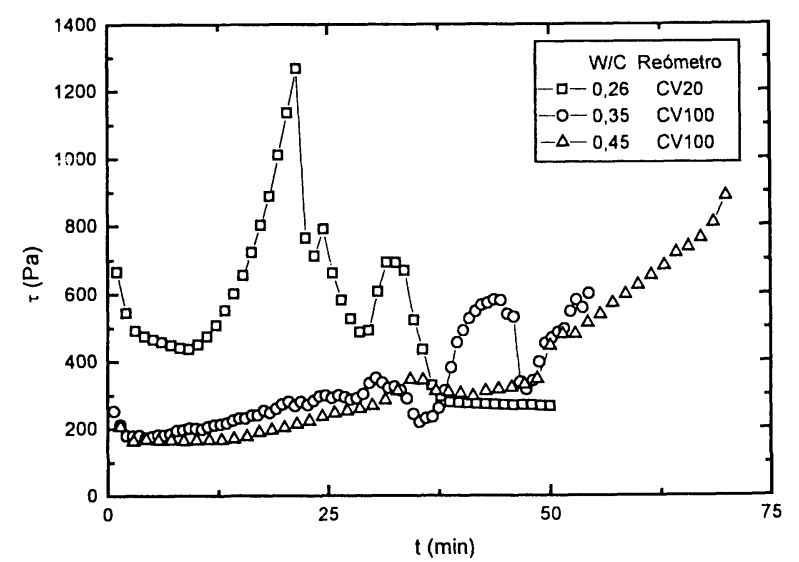

Figura 5.- Evolución del esfuerzo de cizalla con el tiempo para pastas de cemento con diferentes relaciones agua/cemento a velocidad de cizalla constante $(75 \mathrm{rad} / \mathrm{s})$. 
Por otro lado, en la Figura 6 pueden observarse 4 replicados de ensayos a velocidad de cizalla constante sobre la pasta de cemento con relación a/c de 0,26. Es obvio que no es necesario realizar un estudio estadístico para demostrar que este tipo de experiencia tampoco presenta una repetibilidad aceptable con el reómetro CV20.

De igual forma se realizaron estudios estadísticos sobre la repetibilidad de los ensayos sobre las pastas de cemento con relación a/c de 0,35 y 0,45 , llegándose a las mismas conclusiones que con la de 0,26.

Según Cheng (18) esta pobre repetibilidad puede ser debida a los factores siguientes:

-Escasa repetibilidad de la concentración en sólidos y de la distribución de tamaños de partículas en la preparación de las muestras.

-Baja repetibilidad durante el empaquetamiento de las partículas dado que el flujo puede provocar efectos de migración de partículas, rozamiento con las paredes del sistema sensor y floculación de las mismas. Estos efectos dependen de la geometría del sistema sensor $\mathrm{y}$, más concretamente, de la relación:

$$
\mathrm{f}=(\text { diámetro de partícula }) /(\text { hueco de medida })
$$

-Para partículas finas (f pequeña) las medidas reológicas serán independientes del sistema sensor. Sin embargo, si se aumenta el tamaño de partícula puede que diferentes reómetros produzcan diferentes valores. Cuando $f$ aumenta la viscosidad tenderá a valores infinitos debido a que se producen fricciones y atascos en los sistemas sensores.

Un mezclador convencional con control de velocidad de agitación y registro del par torsor, como es el IKA MR$\mathrm{D} 1$, puede ser utilizado como reómetro mediante la

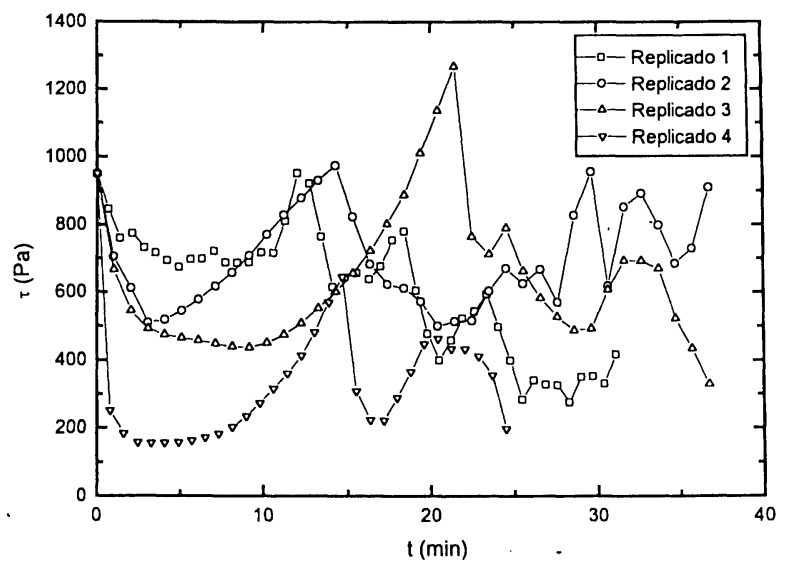

Figura 6.- Evolución del esfuerzo de cizalla con el tiempo para la pasta de cemento con relación agua/cemento de 0,26 a velocidad de cizalla constante $(50 \mathrm{rad} / \mathrm{s})$. aplicación del método de Metzner-Otto (19) que transforma las dos variables anteriores en velocidad de cizalla y viscosidad, respectivamente.

Algunos autores $(20,21,22)$ han demostrado que los agitadores clásicos (turbinas, hélices marinas y anclas) no son eficientes para el mezclado de fluidos newtonianos y no newtonianos altamente viscosos (como es el caso de pastas y morteros de cemento), debido a que se producen efectos de caverna (zonas de bajo mezclado) así como zonas con distinta viscosidad. Por tanto, para obtener un nivel óptimo de mezclado deben utilizarse agitadores en los que se combinen los flujos axial y radial, como pueden ser los agitadores helicoidales, que barren el volumen completo del tanque. Debido a que tanto la pasta de cemento como los morteros son fluidos no newtonianos altamente viscosos se escogió, de entre todos los agitadores disponibles, el agitador de cinta helicoidal.

El método de Metzner-Otto está basado en la analogía de flujo Couette, en la que el flujo producido por un agitador en un tanque puede considerarse similar al producido por un cilindro que gira en el interior de otro (23). Además, introduce el concepto de velocidad de cizalla media, $\gamma_{\mathrm{av}}$, y postula una relación lineal entre dicha velocidad de cizalla media alrededor del agitador y la velocidad rotacional del agitador, $\mathrm{N}$, de la forma:

$$
\gamma_{\mathrm{av}}=\mathrm{k}_{\mathrm{s}} \mathrm{N}
$$

donde $\mathrm{k}_{\mathrm{s}}$ es una constante de proporcionalidad que ha de ser evaluada experimentalmente para cada geometría de interés, y que, en principio, sólo es función de la geometría del sistema. La evaluación experimental de $\mathrm{k}_{\mathrm{s}}$ para el agitador de cinta helicoidal $\left(\mathrm{k}_{\mathrm{s}}=11\right)$ fue llevada a cabo por Cordobés (24).

La curva de flujo de cada sistema se obtuvo mediante el siguiente algoritmo de cálculo:

1) Con cada valor de $\mathrm{N}$ (velocidad de agitación) y de $\mathrm{k}_{\mathrm{s}}$ se obtienen los valores de la velocidad de cizalla media en el tanque, $\gamma_{\mathrm{av}}$.

2) Se calcula el número de potencia, $N_{p}$, mediante la ecuación 3. La relación entre $\mathrm{N}_{\mathrm{p}}$ y el número de Reynolds, $\mathrm{Re}$, en régimen laminar viene dada por la ecuación 4 con la que se calcula este número mediante el valor de la constante de potencia, $\mathrm{k}_{\mathrm{p}}=132$, que fue obtenida por Cordobés para la cinta helicoidal con la que se ha realizado este trabajo.

$$
\begin{gathered}
\mathrm{N}_{\mathrm{p}}=\frac{\mathrm{P}}{\rho \mathrm{d}^{5} \mathrm{~N}^{3}} \\
\text { donde } \mathrm{P}=2 \pi \mathrm{MN} \\
\mathrm{N}_{\mathrm{p}} \operatorname{Re}=\mathrm{k}_{\mathrm{p}}
\end{gathered}
$$


A partir del número de Reynolds se obtiene la viscosidad con la ecuación 5

$$
\operatorname{Re}=\frac{\mathrm{d}^{2} \mathrm{~N} \rho}{\eta}
$$

Tanto la eficacia de este método como la exactitud de los valores de las constantes $\mathrm{k}_{\mathrm{s}} \mathrm{yk}$ fueron contrastadas por Cordobés mediante la concordancia entre los datos de viscosidad de sistemas ternarios líquido-cristalinos laminares, obtenidos mediante los reómetros CV20 y RS100 y los obtenidos con el mezclador convencional IKA MR-D1, con el agitador de cinta helicoidal. Para el caso de pastas de cemento también debería de darse dicha concordancia de datos, aunque, debido a la escasa repetibilidad de las propiedades viscosas, demostrada con los reómetros CV20 y CV100, no es posible verificar este hecho. En la Figura 4 se presentan las curvas de flujo obtenidas con el reómetro CV100 y con el citado mezclador convencional.

Los ensayos realizados en el mezclador convencional mostraron una excelente repetibilidad de las propiedades viscosas de pastas de cemento. En la Figura 7 se representan las curvas de flujo para la pasta de cemento con relación a/c 0,5 , tanto antes como después del ensayo a velocidad de agitación constante. En ambos casos se realizaron barridos de velocidad de ida y vuelta, utilizándose los datos de velocidad de agitación y par torsor de vuelta para obtener la curva de flujo en cada caso mediante el método de Metzner-Otto. Las curvas de flujo obtenidas de esta forma son bastante repetitivas. La media del errorrelativo cometido para cada velocidad de cizalla es de 0,88 y $0,60 \%$ para los ensayos antes y después de la experiencia a velocidad de agitación constante, respectivamente. Esto puede ser debido a que la relación diámetro de partícula/ hueco de medida es muy pequeña, por lo que se evitan los problemas descritos anteriormente. En ambos casos, a las velocidades de cizalla más bajas, se observa el final de la zona pseudoplástica y, posteriormente, una tendencia hacia una viscosidad límite constante a altas velocidades de cizalla, $\eta_{\infty}$

En cuanto al ensayo a velocidad de cizalla constante para la pasta de cemento con relación a/c 0,5 (Figura 8) cabe destacar que la media del error relativo para cada tiempo es de $0,16 \%$. Por otro lado, puede deducirse que el fraguado de dicha pasta comienza, aproximadamente a partir de los 150 minutos, coincidiendo con el valor obtenido mediante la aguja de Vicat.

Después de hallar el sistema más adecuado (IKA MR-D1 con agitador de cinta helicoidal) para la caracterización de pastas de cemento, se trató de obtener las propiedades viscosas de morteros con relación a/c/a de $1 / 2 / 2$, tanto antes como después del inicio del fraguado, así como la evolución de la viscosidad con el tiempo a velocidad de

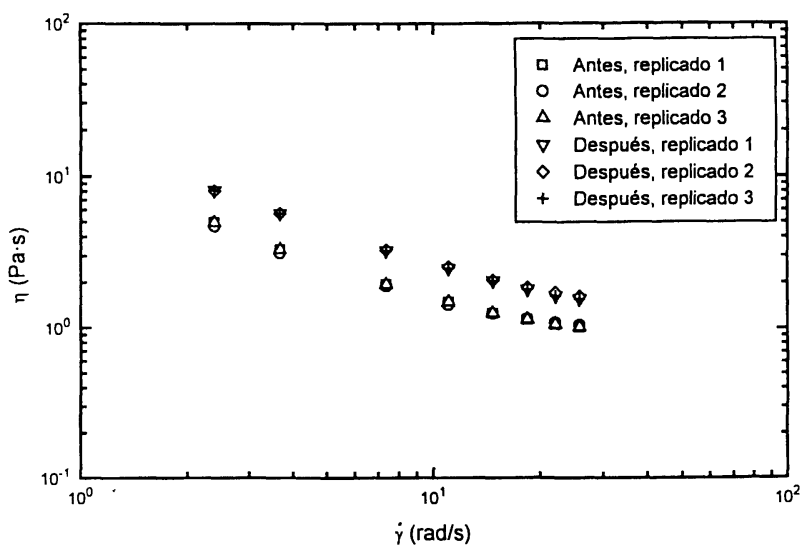

Figura 7.- Curvas de flujo realizadas en el mezclador convencional IKA MR-Dl para pastas de cemento con relación agua/cemento de 0,5 , antes y después del ensayo a velocidad de cizalla constante.

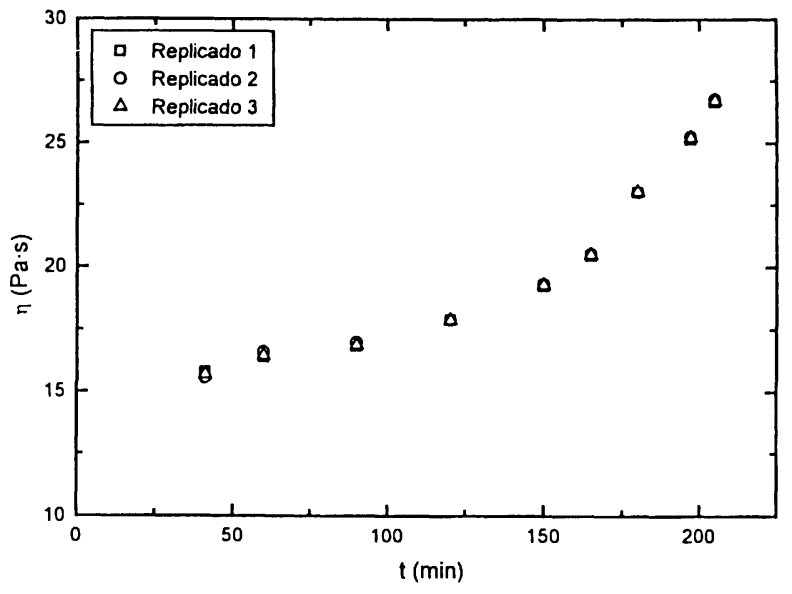

Figura 8.- Evolución de la viscosidad con el tiempo para pastas de cemento con relación de agua/cemento de 0,5 , a velocidad de agitación constante (50 rev./min., IKA MR-D1).

agitación constante. En las Figuras 9 y 11 se observan las curvas de flujo obtenidas de la misma forma que para las pastas de cemento, antes y después del inicio del fraguado, respectivamente, para las diferentes curvas granulométricas. En todas las muestras puede observarse un comportamiento similar a las pastas de cemento y un aumento de la viscosidad de los morteros con la finura de los áridos (25). Por otro lado, los morteros preparados con arena normalizada, que tiene una curva granulométrica bastante similar a la curva intermedia, presentan viscosidades incluso menores que los preparados con la curva inferior. Esto se debe a la mayor esfericidad que presentan los granos de la arena normalizada y a su mayor contenido silíceo que hace que las superficies sean menos rugosas (26).

Por otra parte, en la Figura 10 puede observarse cómo aumenta la viscosidad con el tiempo a una velocidad de agitación constante ( $50 \mathrm{rev} / \mathrm{min}$ ) debido a las reacciones 


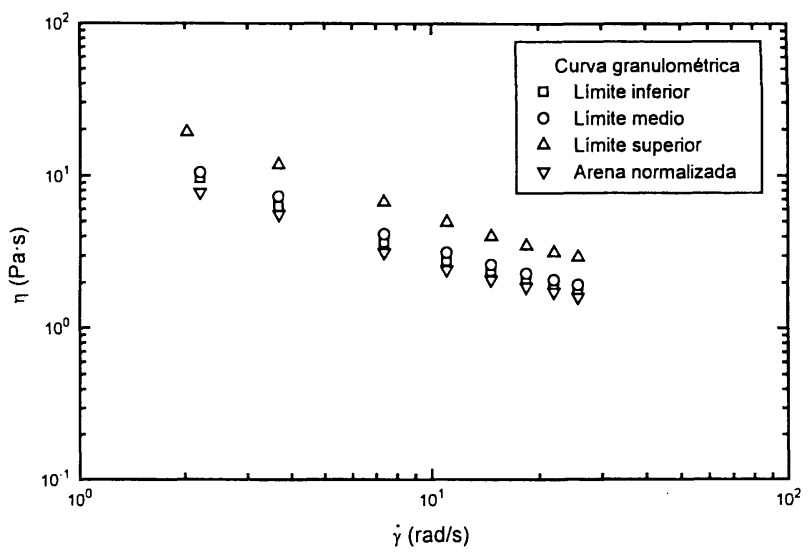

Figura 9.- Curvas de flujo antes del inicio del fraguado para morteros con relación agua/cemento/arena de $1: 2: 2$.

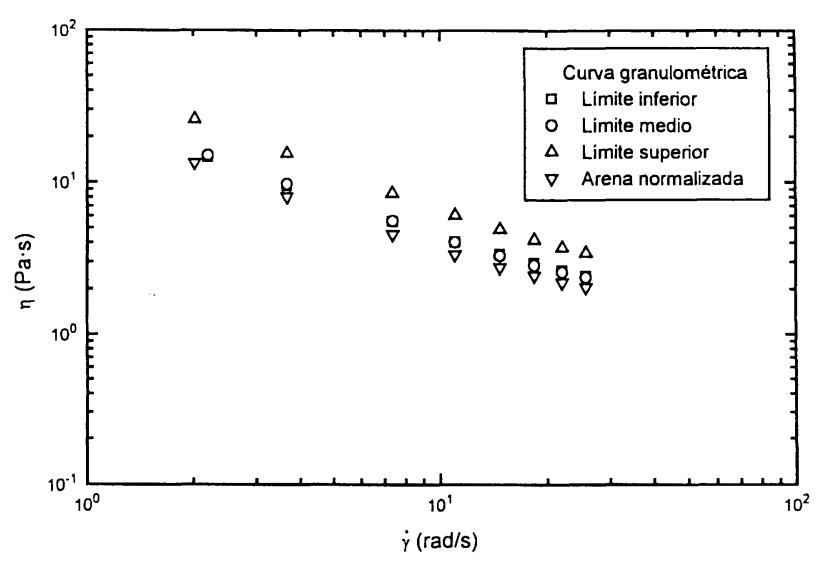

Figura 11.- Curyas de flujo después del inicio del fraguado para morteros con relación agua/cemento/arena de 1:2:2.

de hidratación de los diferentes componentes del cemento. De nuevo vuelve a observarse un aumento de la consistencia con el aumento de la cantidad de finos y el descenso de la esfericidad.

\section{CONCLUSIONES}

Para el estudio reológico de pastas y morteros se ha de utilizar un reómetro que posea: una relación diámetro de partícula/hueco de medida pequeña con el fin de obtener una buena repetibilidad; y el sistema sensor adecuado para que no se den fenómenos de deslizamiento en las paredes de éste. En este trabajo se verifica la eficacia para resolver estos problemas del mezclador convencional IKA MR-D1 con un agitador de cinta helicoidal.

Las pastas de cemento con relaciones a/c de 0,26; 0,35 ; 0,45 y 0,5 presentan un comportamiento tixotrópico y pseudoplástico (entre 0,1 y $10 \mathrm{rad} / \mathrm{s}$ ) con cierta tendencia a alcanzar una viscosidad límite a altas velocidades de cizalla, $\eta_{\infty}$. Aunque, debido a que son suspensiones muy

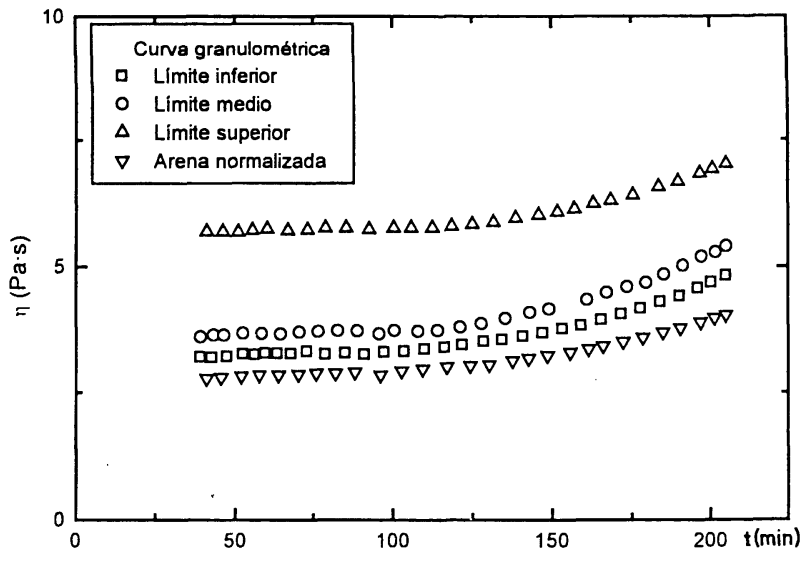

Figura 10.- Evolución de la viscosidad con el tiempo para morteros con relación agua/cemento/arena de 1:2:2, a velocidad de agitación constante (50 rev./min.).

concentradas y a que la relación entre el diámetro de partícula y el hueco de medida en el reómetro es elevada para los reómetros CV20 y CV100, las propiedades viscosas de las pastas de cemento muestran, en este caso, una escasa repetibilidad. Sin embargo, esto no ocurre con el mezclador convencional IKA MR-D1 que ofrece resultados repetitivos. Este mismo tipo de comportamiento, pseudoplástico y tixotrópico, lo presentan los morteros de cemento con una relación a/c/an de 1/2/2.

Debido a que la reología del hormigón está fuertemente influenciada por la de su fase continua (la pasta de cemento o el mortero, según se considere) todos los datos de propiedades reológicas obtenidas en el presente trabajo pueden ser utilizados para obtener el comportamiento viscoso del hormigón mediante correlaciones que han sido desarrolladas por diferentes autores $(27,28,29$, $30,31)$.

\section{AGRADECIMIENTOS}

Los autores agradecen a los laboratorios VORSEVIS.A. por la colaboración prestada en la realización de este trabajo.

\section{BIBLIOGRAFÍA}

(1) Legrand, C.: en La structure des suspensions de ciment et le comportement des suspensions de ciment. Le béton hydraulique, Presses ENPC, Paris, 1982.

(2) Papo, A.: "Rheological models for cement pastes", Materiaux et Constructions, 21 (1988), 41-46.

(3) Caufin, B.; Papo, A.: "Rheological behaviour of cement pastes", Zement-Kalk-Gips, 37(1984)656-661.

(4) Banfill, P.F.G.: "The rheology of fresh mortar", Mag. of Concrete Research, 43, (1991) 13-21.

(5) Costil, V.; Chappuis, J.: "Estude des phénomènes de thixotropie dans les suspensions concentrées de particules minérales", Les cahiers de rhéologie, 13, (1994) 61-70. 
(6) G.Wolter, R.: "Mesuring relative viscosity in cement mortars: mesuring principle and fields of application", Betonwerk-FertigteilTechnik, 12,(1985)816-824.

(7) Tattersall, G.H. y Banfill, P.F.G.: en "The rheology of fresh concrete", Pitman, Londres, 1983.

(8) Brunauer, S.; Kantro, D.L.: "The hydration of tricalcium silicate and dicalcium silicate from 5 to 50 C", en The Chemestry of Cements, Academic Press, 1964, 287-309.

(9) Copeland, L.E.; Kantro, D.L.; "Chemestry of hydration of portland cement at ordinary temperature" en The Chemistry of Cements Vol. I, (HFW Taylor, Ed.), Academic Press, 1964, 313-370.

(10) Le Chatelier, H., Trans. Faraday Soc. 14 (1919) 9.

(11) Ichaelis, W., J. Soc. Chem. Ind. 28 (1909) 836.

(12) Mchedlov-Petrosian: "The formation of cement stone as a theoretical basis of modern concrete technology", VI Siliconf,(1963)315321.

(13) MOPU, Pliego de Instrucción para la Recepción de Cementos (RC-97) Madrid, 1997.

(14) MOPU, NBE FL-90, Muros resistentes de fábrica de ladrillo, Madrid, 1991.

(15) Wang, J-F: "Investigations on a new superplasticizer", Concrete precasting plant and technology, 11 (1995), 98-104.

(16) Lanos, C.; Laquerbe, M.; Casandjianc, C: "Rheological behaviors of mortars and concretes: experimental aproach" en RILEM Proc (Production methods and workability of concrete), E\&FN Spon, vol 32,(1996)343-353.

(17) Banfill, P.F.G.; Carter, R.E.; Weaver P.J.: "Simultaneous rheological and kinetic mesurements on cement pastes", Cement and concrete research, 22,(1991), 1148-1154.

(18) Cheng, D.C.H.; "Further observations on the rheological behaviour of dense suspensions" Powder Technol. 37 (1984), $255-273$.

(19) Metzner, A.B., Otto, R.E.: A.I.Che.E. Journal 3, 1 (1957).

(20) Ulbretch, J.J.; Carreau, P.: en Mixing of Liquids by Mechanical Agitation, (1985).

(21) Skelland, A.H.P.: Non-Newtonian Flow and Heat Transfer, John Wiley, New York, 1967.

(22) Nagata, S.: Mixing-Principles and Applications, John Wiley, Nueva York, 1975.

(23) Brito-de la Fuente, E.: (1992), Tesis Doctoral, Univ. Labal (Quebec).

(24) Cordobés, F.: (1998), Tesis Doctoral, Univ. de Sevilla(Sevilla).

(25) Mewis, J.; Spaull, A.J.B.: Adv. Colloid Interf. Sci., 6(1976) 173-200.

(26) Metzner, A.B. J.: Rheol. 29(1985) 739-775.

(27) Reul, H.: Rheologische Untersuchungen an Zementsuspension mit Flieâ-mitteln. Beton, 1978, H. 10, S. 360-361.

(28) Von Berg, W.: Influence of specific surface and Concentration of solids upon the flow behavior of cement pastes. Magazine of Concrete Research, Vol. 31, No. 109, 179, S. 211.

(29) Wesche, K.; Von Berg, W.: Rheologische Eigenschaften von Zementleim und Frischbeton, Beton, 1973, H. 1, S. 21 -27.

(30) Flatten, H.; Von Berg, W.: Überlegungen zum Verformungsveerhalten von Feinmörtel und Frischbeton. Betonwerk + FertigteilTechnik, 1974, H. 7, S. 469-475.

(31) Wesche, K.; Sybertz, F.: Untersuchungen zum Einfluâ eines Flieâmittels auf die rheologischen Eigenschaften von Portlandzementsuspensionen. Aufgabe zur Diplomarbeit, Institut für Bauforschung der Rheinisch-Westfälischen Technischen Hochschule Aachen, 1981.

\section{publicación del IETCC/CSIC}

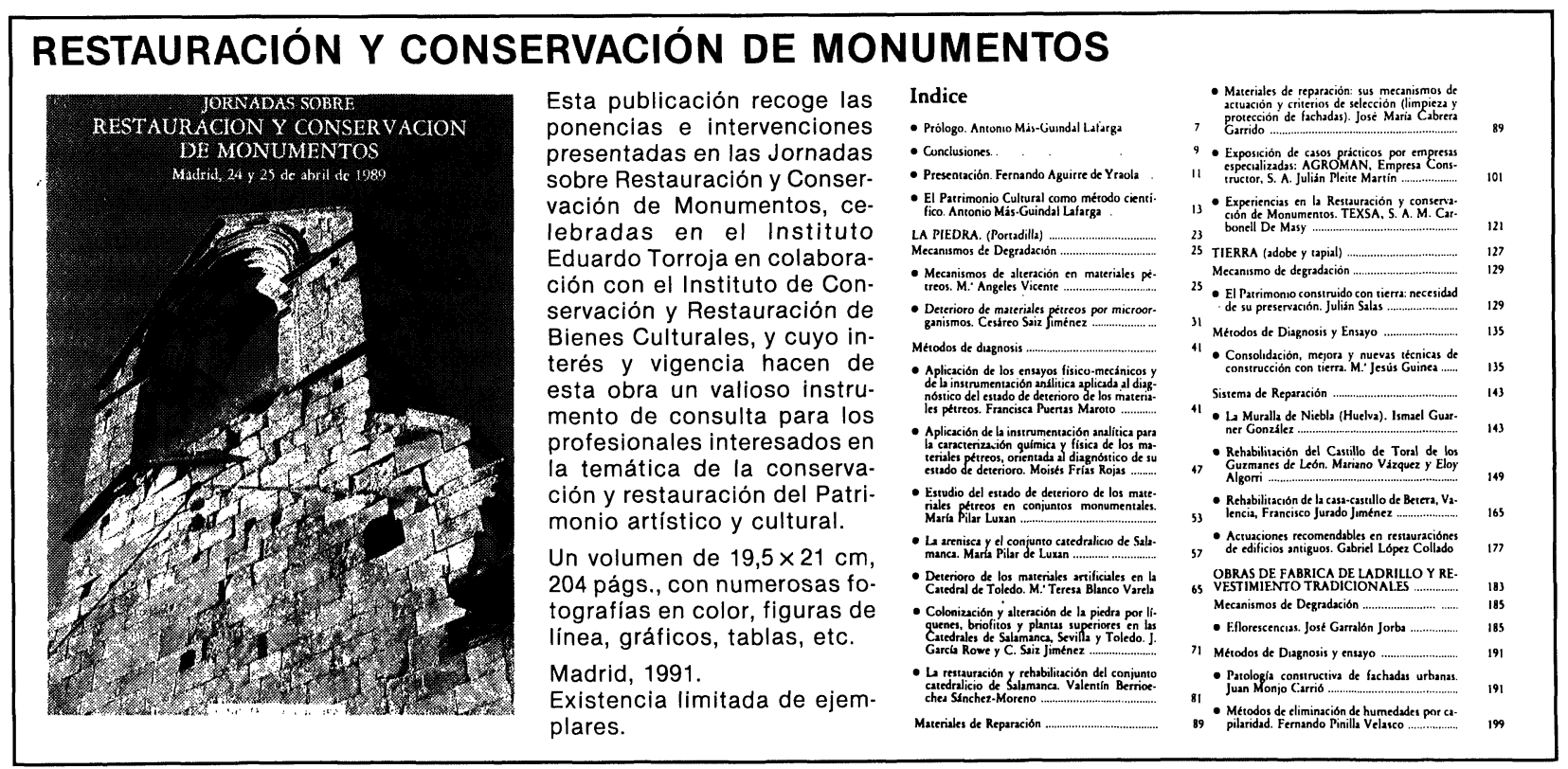




\section{MATERIALES DE CONSTRUCCIÓN}

\section{Monográfico dedicado al VIDRIO EN LA CONSTRUCCIÓN}

La revista MATERIALES DE CONSTRUCCIÓN editada en el Instituto de Ciencias de la Construcción EDUARDO TORROJA, dedica un número doble $\left(\mathrm{N}^{\text {os }} 242 / 243\right)$ a un solo material: El vidrio.

Haciéndose eco del creciente interés que suscitan las investigaciones e innovaciones sobre los productos derivados del vidrio, este material se trata, por primera vez en la Revista, de forma monográfica, con la amplitud que permite un número especial.

Los distintos artículos que componen este número son muestra de la gran variedad de características, propiedades y aplicaciones bajo las que se presentan los derivados del vidrio. Entre sus usos más comunes se encuentran: elementos transparentes de cerramientos, materiales estructurales en las fachadas, aislamientos de muros y cubiertas, recubrimientos de pisos y paredes, componentes de refuerzo de placas, paneles, morteros y hormigones, como material decorativo y estético. Hoy en día, el vidrio es un material indispensable en laconstrucción y su tecnología está en continua evolución para dar respuesta a la constante demanda de nuevos productos con carácter multifuncional.

En este númeroespecial se recogen trabajos de investigación originales e inéditos que cubren una temática muy diversa dentro de los materiales vítreos destinados o aplicados en construcción. Aparecen artículos relacionados con las vidrieras artisticas, los acristalamientos, los vidrios funcionales, las fibras de refuerzo, los vitrocerámicos y los porcelanatos. Los autores de estos trabajos, tanto los nacionales comolos internacionales, son especialistas reconocidos en sus respectivos campos científicos

Los artículos que componen este número especial son:

- Procesos de alteración de las vidrieras medievales. Estudio y tratamientos de protección.

(Alteration processes of medieval stained glass windows. Studl and protection treatments).

J. $M^{\mathrm{a}}$ Fernández Navarro.

- El efecto de la corrosión en vidrieras coloreadas.

(Ihe effect of corrosion of stained glass windows).

J. Leissner.

- Formulación de vidrios absorbentes del calor

(Formulation of heat absorbing glasses).

P. Álvarez-Casariego y P. Mazón.

- Dimensionamiento de placas de acristalamiento para edificios mediante un modelo probabilístico de rotura.

(A probabilistic model for failure design of glass plates in building).

A. Fernández Canteli, I. Viña y A. Bernardo Sánchez.

- Caracterización de fibras en forma de lana de roca para aislamiento obtenidas a partir de basaltos canarios.

(Characterization of fibers as rockwool for insulation obtained from canary islands basalts).

J. M. Cáceres, J. E. García Hernández y J. Man Rincón.

- El GRC: Material compuesto de matriz inorgánica reforzado con fibras de vidrio AR.

(GRC: Composite material from an inorganic matrix reinforced with ar glass fibres).

P. I. Comino.

- Los materiales vitrocerámicos en la construcción.

(Glass-ceramic as building materials).

J. M Rincón y M. Romero.

:- Gres porcelánico: Aplicaciones arquitectónicas, procesado y propiedades físico-mecánicas.

(Porcelainized stoneware: Architectural, processing and physico-mechanical properties).

T. Manfredini, M. Romagnoli y J. Ma Rincón.

Venta de ejemplares: Distribución de Publicaciones

Instituto de Ciencias de la Construcción Eduardo Torroja

Serrano Galvache, s/n - 28033 Madrid.

Tfno.: (91) 302.04.40 - Fax: (91) 302.07.00 